$37.6 \mathrm{~g} / \mathrm{L}$ vs $38.4 \mathrm{~g} / \mathrm{L}$ for the overall study group $(\mathrm{p}=0.81$, NS).

Conclusions Upper GI mural thickening on CT cannot be dismissed. Despite oesophagitis, gastritis and hiatus hernia making up most endoscopic diagnoses (75\%), it correlated with malignancy, dysplasia or metaplasia in $10 / 59(17 \%)$ patients in this study. Patients with malignancy could not be accurately differentiated by indication for imaging or by biochemical markers. We conclude that there is good concordance in pathology detection at gastroscopy following findings of thickening on CT scan. We recommend gastroscopy is performed in every case when this abnormality is detected incidentally.

\section{PTU-071 HELICOBACTER PYLORI BREXIT: NICE VS MAASTRICHT A COMPARISON OF ERADICATION GUIDELINES AND RESISTANCE IN LONDON}

Cheh Kuan Tai ${ }^{*}$, Annette Jepson, Laura Marelli. Homerton University Hospital NHS Foundation Trust, London, UK

\subsection{6/gutjnl-2019-BSGAbstracts.287}

Introduction The Public Health England (PHE) advises HP testing and treatment if positive, in patients without alarm symptoms who have uncomplicated dyspepsia unresponsive to lifestyle change and antacids. However, the suggested antibiotic therapy is incongruent with latest Maastricht V/Florence guidelines which suggest bismuth containing quadruple therapy first line in regions with high metronidazole and clarithromycin resistance and second line routinely. ${ }^{1}$ In London, a study showed overall resistance to metronidazole at 59\% and clarithromycin at $11 \%$ with non-UK birth being main risk factor. ${ }^{2}$ Our trust serves a local population where $31 \%$ consist of black and ethnic minorities. We aimed to look at our local $\mathrm{HP}$ resistance data and compare the antibiotics regimes used in different trusts within London.

Methods A list of all gastroscopies with CLO testing over a year between October 2017-18 was generated.

A list of all HP isolates sent to the microbiology lab over a 2-year period from January 2016 to December 2018 was generated.

We performed a search on the 'Microguide' app for HP antibiotic regimes for trusts within London.

Results Between October 2017 and September 2018, the endoscopy unit performed 1375 CLO tests out of 5000 gastroscopies. A review of 100 patients who had CLO testing showed that they were all appropriate.

Between 2016 and 2018, 36 isolates of HP were cultured. 35 were resistant to metronidazole, 26 to clarithromycin, 10 to levofloxacin, 1 to tetracycline and none to amoxicillin.

We found antibiotics guidelines for 6 London trusts on 'Microguide'. None used antibiotic duration of 10-14 days and only 1 out of 6 recommended quadruple bismuth therapy as second line treatment.

Conclusion Patients in whom we send HP resistance testing have high resistance rates to conventional first line antibiotics. Given that these patients are likely to have previously failed at least one treatment regime; the results are inevitable skewed towards resistant isolates. While NICE and PHE guidelines are appropriate for some populations, areas of London which are at risk of higher resistance rates should use Maastricht guidelines. Therefore, we recommend a 10-day course of bismuth-containing quadruple therapy as second line.

\section{REFERENCES}

1. Malfertheiner P, Megraud F, O'Morain CA on behalf of the European Helicobacter and Microbiota Study Group and Consensus, et al. Management of Helicobacter pylori infection-the Maastricht V/Florence Consensus Report. Gut 2017;66:6-30.

2. Elviss N, Owen R, Breathnach A, Palmer C, Shetty N. Helicobacter pylori antibiotic-resistance patterns and risk factors in adult dyspeptic patients from ethnically diverse populations in central and south London during 2000. Journal of Medical Microbiology 54:567-574.

\section{PTU-072 A MULTI-CENTRE REVIEW OF ACUTE UPPER GI BLEEDING; CAN BLOOD UREA LEVELS AID DIAGNOSIS?}

${ }^{1}$ Deevia Kotecha, ${ }^{2}$ Jason Mak, ${ }^{2}$ Neel Sharma, ${ }^{2}$ Ralph Boulton, ${ }^{3}$ Benjamin Disney, ${ }^{2}$ Neeraj Bhala, ${ }^{1}$ Ajay Verma*. ${ }^{1}$ Kettering General Hospital NHS FT, UK; ${ }^{2}$ University Hospitals Birmingham NHS FT, UK; ${ }^{3}$ University Hospital Coventry and Warwickshire NHS FT, UK

\subsection{6/gutjnl-2019-BSGAbstracts.288}

Introduction Risk scoring for acute upper gastrointestinal bleeding (AUGIB) is key when assessing patients for requiring OGD. The Rockall score utilises age, comorbidities and shock. The Glasgow-Blatchford score (GBS), in addition, utilises haemoglobin, melaena, and blood urea levels.

Raised blood urea levels can represent digestion of blood from the upper gastrointestinal tract giving rise to melaena; the presence of both gives a high GBS. However, inexperienced health care professionals can misinterpret the absence/ presence of melaena, raised urea levels may be due to kidney injury. Nevertheless, Gastroenterologists may use urea to diagnose AUGIB if patients haven't had overt/witnessed/reliably reported haematemesis or melaena.

It has been shown that a raised urea:creatinine ratio (URCR) can be associated with AUGIB and may be superior to urea alone as it mitigates for kidney injury. ${ }^{1}$ However, URCR is not widely used in the UK in the assessment of AUGIB. We aim to assess the association of urea and URCR levels with AUGIB.

Methods A retrospective review at three UK centres (Kettering General Hospital, Queen Elizabeth Hospital Birmingham, and University Hospital Coventry \& Warwickshire) was undertaken. Endoscopy reports and blood tests were reviewed of patients undergoing inpatient OGD for suspected AUGIB within 2017/8, data were recorded in an Excel spreadsheet. URCR was calculated by dividing Urea by creatinine, and multiplying by 1000 (abnormal $=\geq 100$ ). Statistics were analysed using SPSS.

Results 357 patients' records were reviewed (median age = 68), 179 had a plausible AUGIB (50.1\%). Receiver operator characteristic (ROC) curves for Urea gave an area under the curve $($ AUC $)=0.733$. For URCR, AUC $=0.789$.

Binary logistic regression modelling was performed using age, urea, and URCR. $\chi^{2}(3, \mathrm{n}=357)=102.92, \mathrm{p}<0.001$. $25-34 \%$ of the variance in AUGIB is explained by the model.

The model URCR value of 97.7 can be used to predict AUGIB, applying this to our data set correctly identifies 124/ 179 patients with AUGIB (69.3\%), and is predicted to correctly identify $74.5 \%$

Conclusion This pilot study has limitations as bleeding lesions may have not been identified at OGD. Urea and URCR have AUCs of $0.733, \& 0.789$. Logistic regression modelling suggests a URCR level of 100 would correctly identify $\sim 70 \%$ of AUGIB in patients with suggestive symptoms. Outside of firm 